University of Nebraska - Lincoln

DigitalCommons@University of Nebraska - Lincoln

Educational Psychology Papers and

Publications

Educational Psychology, Department of

January 1997

Conceptual and Empirical Bases of Conjoint Behavioral

Consultation

Susan M. Sheridan

University of Nebraska-Lincoln, ssheridan2@unl.edu

Follow this and additional works at: https://digitalcommons.unl.edu/edpsychpapers

Part of the Educational Psychology Commons

Sheridan, Susan M., "Conceptual and Empirical Bases of Conjoint Behavioral Consultation" (1997). Educational Psychology Papers and Publications. 59.

https://digitalcommons.unl.edu/edpsychpapers/59

This Article is brought to you for free and open access by the Educational Psychology, Department of at DigitalCommons@University of Nebraska - Lincoln. It has been accepted for inclusion in Educational Psychology Papers and Publications by an authorized administrator of DigitalCommons@University of Nebraska - Lincoln. 
Published in School Psychology Quarterly, 12:2 (1997), pp. 119-133. Copyright (C) 1997 American Psychological Association. Used by permission. "This article may not exactly replicate the final version published in the APA journal. It is not the copy of record." http://www.apa.org/journals/spq/

Accepted October, 1, 1996. Action Editor for School Psychology Quarterly: Joseph C. Witt.

Portions of this article were presented as an invited address at the 1994 American Psychological Association annual conference. The article was presented in acceptance of the Lightner Witmer Award for early career contributions, bestowed to the author by Division 16 (School Psychology) of the $A P A$.

\title{
Conceptual and Empirical Bases of Conjoint Behavioral Consultation
}

\author{
Susan M. Sheridan \\ University of Utah
}

\begin{abstract}
This article describes "conjoint behavioral consultation," a model of service delivery that addresses the shared responsibility among school personnel, families, and other important systems in educational problem solving. The conceptual background (incorporating ecological-systems and behavioral theories) and important features of the model are reviewed briefly. Findings from a number of empirical studies, including outcome studies, case studies, process research, and acceptability research, are reviewed. Directions for future research are also explored.
\end{abstract}

The literature has convincingly assured us over the last decade of the strong, positive relationship between parental involvement/home-school partnerships and a child's success. Even before the influx of attention given this topic, I believed in the importance of such collaborations.

I worked for some time as a school psychologist in a small, blue-collar community. Throughout the course of psychoeducational evaluations, I met with many parents and began seeing firsthand the relationship between a child's family life and his or her achievement at school. Some parents with whom I worked were truly committed to working with school personnel to enhance their child's school success. Others showed no interest at all, and it was often questioned whether they even knew where the school was located. Sadly, many students with whom I worked came to school unfed, dirty, and tired. Some complained of being kept up by their parents' late parties, and others reported that they had completed their homework by candlelight in the lounge at the local bowling alley. One boy, whom I still think about today, was sexually abused by an older 
brother with a knife held at his throat. It's no wonder these children were preoccupied with thoughts unrelated to learning while at school.

Being in a role that required a great deal of assessment, I often felt frustrated that I could not do more for students like these who could be considered "at risk." I began perceiving the assessment role as extremely limiting, and did not feel as though there was a relationship between standardized test results and meaningful interventions. IQ scores and achievement levels led to educational classifications, which led to placement into a resource classroom (which seemed to happen automatically, although that was not the intent of the special education law). Special education (especially in part-time resource programs) seemed to be simply a, place where students could go for a specified period of time; in most cases, it was not individualized intervention. When students did not qualify, teachers usually expressed varying degrees of dissatisfaction but rarely wanted to do anything differently themselves, in their own classrooms. Yet the importance of a child's home life in his or her educational success kept gnawing at me.

My frustrations with assessment and placement could have been addressed if only I had known more about consultation. Consultation-based services are in some ways philosophically different than those based on standardized tests that yield standard scores, percentiles, and grade equivalents. Rather than asking about a student's IQ or ranking in comparison to others of the same age, consultation services are based on questions regarding what a student can and cannot do (i.e., his/ her functional skills) in a certain domain, what events in the environment may be contributing to a student's difficulties, and what environmental factors can be altered to enhance a student's skill development. Rather than seeking to identify a pathological condition within a child, behavioral consultants seek to understand what function certain behaviors are serving. Conditions surrounding the behavior can then be altered so that needs are met appropriately and effectively. Although it is important to know that a child has a diagnostic condition such as depression, it is at least equally important to know how the depression manifests itself and what events trigger or maintain depressive episodes. These behavioral manifestations and environmental events can then be targeted for intervention.

Behavioral consultation research and practice has focused almost solely on students' problems as they are manifested in school settings, with little recognition of the other settings and systems within which children function. The research on home-school collaboration has clearly demonstrated that parental involvement in education has positive and unequivocal effects for students, families, teachers, and schools (Christenson, 1995). Clearly, there is a need to engage parents constructively and meaningfully in educational planning. Simultaneous consultation with parents and teachers is one way to facilitate collaborative partnerships among significant individuals in a child's life. Conjoint behavioral consultation represents an effective marriage between the empirically-validated structured approach of behavioral consultation, and the important conceptual advances in the area of home-school partnerships. 


\section{CONJOINT BEHAVIORAL CONSULTATION}

Conjoint behavioral consultation $(\mathrm{CBC})$ is a conceptual and practical extension of a traditional approach to behavioral consultation. In CBC, a consultant works with a teacher or parent simultaneously and interdependently rather than in a parallel manner. Conjoint behavioral consultation is defined as a structured, indirect form of service-delivery, in which parents and teachers are joined to work together to address the academic, social, or behavioral needs of an individual for whom both parties bear some responsibility (Sheridan \& Kratochwill, 1992; Sheridan, Kratochwill, \& Bergan, 1996). It is designed to engage significant consultees from various systems in a collaborative problem-solving process.

\section{Conceptual Bases}

In conjoint behavioral consultation, the interconnections between the various systems in a child's life are recognized as critically important. The model is based on an integration of ecological-systems and behavioral theories, which in combination address both systemic (home, school, and other) influences and functional (antecedent, consequent, and setting) events.

Ecological-systems theory is concerned with the child as part of a system or network of subsystems. Accordingly, a child's "problem" does not reside within the child, nor exclusively in his/her environment. Rather, a behavior occurs as a function of the interaction of the child with the systems of which (s)he is a part. The parts of a system are interconnected, and change in one part of a system affects other system components.

Central to ecological-systems theory is the assumption that each child is an inseparable part of a small social system. Further, children transcend systems (e.g., home, school, and peer groups), and become involved in several systems. The systems overlap, and what occurs in one system affects the child's behaviors in other systems. Beyond the immediate setting, however, the theory recognizes the importance of the interrelations and linkages among the major settings and systems in one's life (e.g., interactions between the home and school, or between the child's parents and peer group). The interconnections between systems presumably have a significant impact on development. Just as influences within families and schools are reciprocal, influences between them are reciprocal; a child's school experiences influence his/her experiences and behavior at home, and family experiences influence school behavior and performance (Conoley, 1987; Hansen, 1986). Therefore, from an ecological-systems perspective, a consultant should strive to understand the network of interrelationships among various individuals and their environment (Apter, 1982).

Although some guidelines are provided for the clinician who utilizes an ecological-systems approach, the procedures are generally descriptive and nonstandardized. This format may present difficulties for the psychologist working with the family and school collaboratively, in that specific roles and respon- 
sibilities are less well-defined, and specific problem-solving procedures may be complex and ambiguous. While most therapists conclude that their methods are effective, specific procedural information is lacking. This issue becomes particularly problematic when replication in research is attempted.

To promote effective home-school partnerships, clear and operational procedures are necessary to guide one through the process of implementing services. Unfortunately, such procedural guidelines are unavailable in the ecological-systems theory literature. Further, little empirical evidence exists that articulates the efficacy of the theory in consultation. Therefore, ecological-systems provides a useful framework to guide conceptualization of a broader consultation approach. Behavioral theory, and behavioral consultation in particular, provides a useful, empirically documented framework for working within and between systems to effect change (Bergan \& Kratochwill, 1990; Kratochwill \& Bergan, 1990). The structured problem-solving approach is particularly effective in linking parent and school resources in a conjoint, collaborative process.

Earlier conceptions of the CBC model included two significant systems in a child's life — home and school (cf. Sheridan \& Kratochwill, 1992; Sheridan, Kratochwill, \& Elliott, 1990). However, it is now recognized that many other systems beyond the home and the school interact in the life of a child, and should be included in a comprehensive and consistent plan. These may include extended family members, alternative caregivers, clergy, support service personnel, probation officers, therapists, or a host of others who have regular and meaningful contact with the child. Such individuals may provide additional information about the problem, as well as meaningful observations that can contribute to an accurate functional analysis. Further, they can serve as additional resources when delineating intervention strategies and tactics. Even persons within the school can be included as additional support systems if they have a meaningful and ongoing relationship with the child (e.g., school counselors or social workers). Figure 1 depicts a conceptual framework for $\mathrm{CBC}$, emphasizing the reciprocal, interacting, and expanded systems in a child's life.

Conceptually, CBC focuses on the interacting systems within a child's life. Practically, a simultaneous, conjoint model extends earlier consultation work by providing services to parents and teachers together. By actively joining parents and teachers in a structured problem-solving framework, comprehensive and systematic data can be collected on the child's behavior over time and across settings. Data collection over time and across settings may help identify various events that may be functionally related to, but removed in time and place from target behaviors (i.e., setting events; Wahler \& Fox, 1981). For example, influences at home such as an unstructured or chaotic morning routine may influence a child's behaviors in the classroom, just as conflicts with a teacher or peer at school may affect a child's interactions with family members at home.

Consistent programming across settings is an important element in behavioral therapy, and is a central feature of CBC. Implementation of structured and con- 


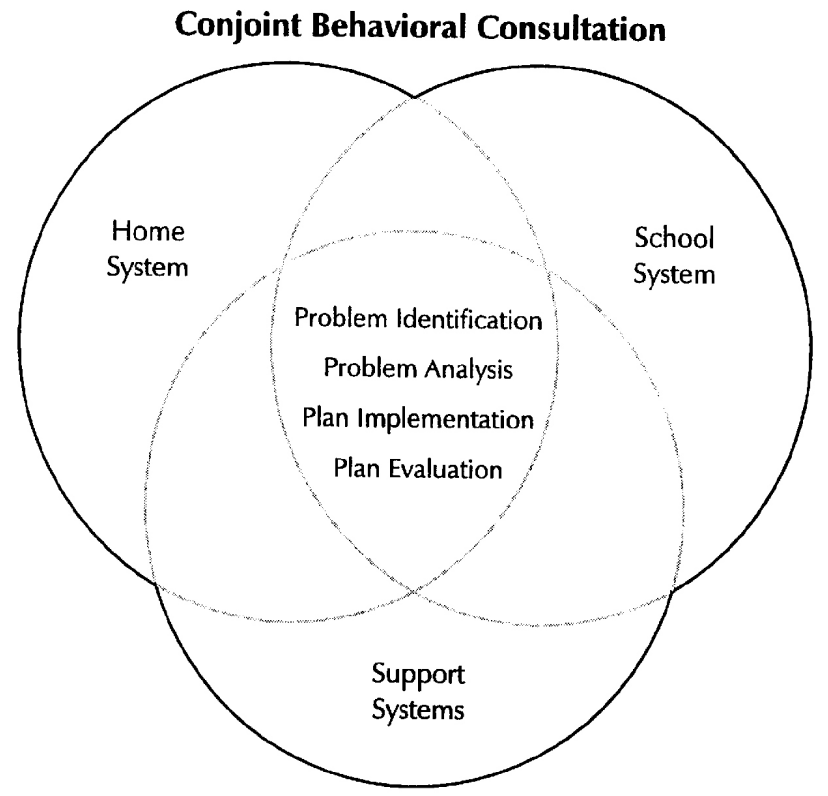

Figure 1. Conceptual framework for conjoint behavioral consultation. [Reproduced with permission from Sheridan, S. M., Kratochwill, T. R., \& Bergan, J. R. (1996). Conjoint behavioral consultation: A procedural manual. New York: Plenum.]

stant contingencies in various settings enhances generalization and maintenance of consultation treatment effects (Drabman, Hammer, \& Rosenbaum, 1979). Finally, involving significant treatment agents across settings can help monitor the occurrence of behavioral side effects (undesired intervention effects that are unplanned or unexpected) and contrast effects (effects in nontreatment settings or conditions that run counter to those under treatment conditions). The assessment of such effects can occur readily since individuals from various settings are involved in conjoint consultation. Modifications in the program can be made immediately if such effects are observed.

Goals in conjoint behavioral consultation encompass both process and outcome issues. It is hoped that by involving family members in consultation, communication with and knowledge about the child's home system can be increased. Enhancing the home-school relationship is a central goal of CBC, with emphasis on empowering parents to become an active and meaningful part of problem solving. Likewise, it is expected that by involving more individuals in assessment and intervention, the diversity of expertise and resources available for problem conceptualization and solution will be increased.

Outcome goals in CBC relate to both client and consultee competencies. Specifically, a primary goal is to effectively resolve client difficulties that are being addressed in CBC. The skills and behaviors of clients and consultees may be en- 
hanced through the problem solving process. Generalization and maintenance of treatment effects through consistent intervention programming is an additional outcome goal. Likewise, it is hoped that further conjoint problem solving among consultees can occur following the resolution of a particular target problem.

\section{Empirical Bases}

The empirical base for $\mathrm{CBC}$ is expanding. Empirical investigations and structured case studies in conjoint behavioral consultation have been conducted to evaluate the efficacy of the model for various target problems. Some preliminary research has also been conducted assessing the communication processes that have been identified in successful conjoint consultation cases. Likewise, one study evaluated a national sample of school psychologists' reported acceptability of the model.

Outcome Research. Outcome research assessing the effectiveness of conjoint behavioral consultation is accumulating. In an early study Sheridan et al. (1990) were concerned with increasing the social initiation behaviors of socially withdrawn children. Of particular interest was the demonstration of behavioral generalization to the home setting. The participants in this study included four socially withdrawn children from a rural town in the midwest (three girls, one boy; ages 8-12). Selection criteria included teacher referral, low scores on the Social Initiation subscale of a prepublication version of the Social Skills Rating System (SSRS; Gresham \& Elliott, 1990), direct observational data indicating low levels of social initiations toward peers, and parent and teacher interview data. There were two treatment conditions in the study (i.e., $\mathrm{CBC}$ and consultation with teachers only). In the $\mathrm{CBC}$ condition, teachers and parents worked together with a school psychologist consultant; in the teacher-only condition, parents were not included in consultation. In both experimental conditions, children were exposed to the same behavioral treatment (i.e., goal setting, self-monitoring, and positive reinforcement). In the $\mathrm{CBC}$ condition, these same procedures were implemented across home and school settings. In teacher-only consultation, they were instituted at school only. Multiple baseline across subjects designs were used to evaluate the effectiveness of the separate consultation interventions.

Baseline performance for each child was approximately 1 initiation per week at school; during the last phase of treatment, performances increased to between 30 and 40 initiations per week. At home, baseline levels ranged from approximately 1 initiation per week, to approximately 7 per week during the last phase of treatment. When consultation was undertaken with parents and teachers together, initiations increased in both home and school settings. However, when consultation was undertaken with teachers only, children's initiations increased at school only. Baseline performance for each child was 0 to 1 initiation per week at school, with weekly rates of between 6 and 26 during the last phase of treatment. At home, baseline rates were between 0 and 1.5 per week, increasing to between only 1 and 4 per week during the last phase of treatment. Treatment gains 
at school were maintained for all children in both conditions, but were most notable for those in the CBC condition.

In summary, this study was the first to investigate the efficacy of CBC. In general, the traditional use of behavioral consultation with teachers was found to be effective in increasing the social initiation behaviors of socially withdrawn children at school. However, generalization of treatment effects to the home setting was demonstrated only when parents were actively involved in consultation and treatment. Maintenance of treatment effects also appeared to be stronger when conjoint consultation procedures were utilized. Social validity and treatment integrity measures were also included and yielded positive results.

Colton and Sheridan (in press) furthered $\mathrm{CBC}$ research by demonstrating its utility for a limited number of students with ADHD experiencing difficulties with social interactions. Thus, it tested the model with a new behavioral target and diagnostic group and involved parents from low SES conditions as consultees. This outcome study combined $\mathrm{CBC}$ with a behavioral social skills training and reinforcement program to address social performance deficits in cooperative play behavior. Subjects were three boys (ages 8-9) diagnosed with ADHD, their mothers living in low socioeconomic conditions, and their teachers (second and third grades).

The behavioral social skills treatment program consisted of four major components: (a) social skills coaching and role play; (b) a home-school communication system; (c) self-monitoring of recess behaviors; and (d) positive reinforcement. In general, each parent and teacher dyad jointly selected seven social skills, each of which represented a form of "cooperative play" (e.g., playing a game, problem solving, responding to teasing). These skills served as direct targets of training. The steps for each skill (adapted from McGinnis \& Goldstein, 1984) were written on "Friendship Recipe Cards" which served as a medium for coaching. Coaching instructions were included with each "Friendship Recipe Card" to instruct parents and teachers in the appropriate procedures, and to increase treatment integrity.

After the participant was coached in the skill identified on the Friendship Recipe Card he was responsible for practicing the skill, self-monitoring his performance on the playground, discussing it with his teacher and completing a home-note, and receiving positive reinforcement at home. The home-based reinforcer was delivered contingent upon the subject practicing the "friendship skill recipes," discussing his performance with his parents, and returning the home note to school.

Outcome measures in this study included direct observations of positive interaction behaviors in semi-naturalistic play settings, and behavioral rating scales. Specifically, 20-minute direct (partial interval) observations were conducted one to two times weekly in analogue play situations to assess participants' positive interaction behaviors toward classmates. The play-based observation sessions were videotaped and observed by two blind, independent trained observers in random order, who also coded $33 \%$ of the same tapes for purposes 
of interrater reliability (overall, 92\% agreement was achieved). The Social Skills Rating System-Teacher, Parent, and Student forms (Gresham \& Elliott, 1990) were also used for purposes of assessing child outcome.

Social validity was assessed using social comparison and subjective evaluation procedures (Kazdin, 1977). Parents' and teachers' acceptability of CBC was assessed on the Behavior Intervention Rating System (Von Brock \& Elliott, 1987). Child-subjects' acceptability of the treatment protocol was assessed by the Children's Intervention Rating Profile (CIRP; Witt \& Elliott, 1985). Treatment integrity was assessed for both conjoint behavioral consultation procedures and for parents' and teachers' utilization of treatment procedures, and both were determined to be very high.

A multiple baseline across subjects design was used to evaluate the effects of the $\mathrm{CBC} /$ social skills treatment program on target children's cooperative play behaviors. All subjects increased positive play behaviors with peers during treatment phases. Data trends suggested that the $\mathrm{CBC} /$ social skills program exerted functional control for two of the subjects. On the other hand, data for the third child were variable with a high degree of overlap, suggesting little experimental control. All parent and teacher ratings on the SSRS (with the exception of the parent rating for Child 1) indicated positive increases in overall social skills scores from pre- to post-treatment.

Social comparison data suggested that all children demonstrated substantial behavioral gains and increased their positive interactions that approached the level of "normal" comparison peers. Further, parents and teachers reported that the procedures were acceptable, and child responses suggested that they found the social skills intervention highly acceptable.

Case Studies. Some conjoint behavioral consultation case studies have also been conducted. In an extensive carefully controlled set of case studies, the model was evaluated with academically underachieving children (Galloway \& Sheridan, 1994). Participants were six primary grade students (grades 1-3) who often failed to complete math assignments on time, and/or with acceptable levels of accuracy. They all demonstrated performance deficits; they had the skills to complete assignments with accuracy, but often failed to do so. Likewise, the children demonstrated variable performance that was resistant to previous documented intervention attempts.

In two separate sets of case studies, the investigators evaluated the effectiveness of a standard intervention with and without the inclusion of CBC. Both studies involved the use of a home-note, wherein teachers recorded subjects' daily performances in math, as well as process behaviors intended to help them complete work (e.g., pencil ready, papers out). The home note also included a checklist to help remind parents of what to do at home, and served as a measure of treatment integrity.

A manual was developed that instructed parents in the use of the home note, including potential reinforcers and ways to handle problems. In the first set of case studies (home-note only), the manual and home note served as the only interven- 
tions. In the second, the home note and manual were used, but were instituted in the context of conjoint behavioral consultation. Students were also involved in the latter part of consultation (plan implementation and treatment evaluation).

The investigators used $\mathrm{AB}$ with replication designs to assess outcomes of the home note and conjoint behavioral consultation interventions. All three children in the home note only case studies showed mean improvements in math completion and accuracy from baseline to treatment conditions. For subjects in these case studies, however, the data continued to be extremely variable during the intervention phase.

As in the home note condition, all children in the home note with $\mathrm{CBC}$ condition demonstrated improvements in math completion and accuracy, but the gains were greater and more stable. Although baseline data were variable for both completion and accuracy, the improved performance during treatment was considered stable for all three children. Findings in the CBC case studies also suggested enhanced treatment integrity, maintenance of treatment gains at follow-up and consumer acceptability. Furthermore, parents in the CBC case studies adhered more faithfully to the treatment regimen than did parents in the home-note only case studies, which may be one reason for the greater treatment effects. Parents in the $\mathrm{CBC}$ case studies also used home reinforcers more effectively than did parents in the home note only condition. Teachers in the $\mathrm{CBC}$ condition reported greater satisfaction; however, there was no difference in the degree of treatment integrity demonstrated by teachers.

In sum, for students with a history of social and academic performance problems who had not responded to traditional intervention strategies, the addition of consultation with parents and teachers appeared to have enhanced treatment outcomes. Maintenance and generalization of treatment gains appeared to be stronger when more intensive consultation procedures were utilized (i.e., when parents were actively involved in consultation and treatment). Based on the findings of Galloway and Sheridan (1994), this does not appear to be due simply to the provision of information. It is possible that the improvement factors may be related to the relationship that develops among participants in the CBC process; however, this is an empirical question in need of research attention.

Another case study involved a child with irrational fears (Sheridan \& Colton, 1994). Specifically, a Kindergarten teacher referred a six-year-old boy who spoke of nightmares repeatedly in school. The boy reported vivid stories of monsters and spiders in his room who often grabbed him at the ankle. He was terrified of sleeping alone, and as a result, slept in his parents' room all night, every night more than two years. The goal for consultation in this case was to get the child to sleep in his own room on a consistent basis.

Treatment involved a fading of environment and positive reinforcement procedure, wherein positive reinforcers were delivered each time the child slept in a spot that moved successively closer to his own room. An AB case study design was used, with the criterion being adequate performance demonstrated over two occasions at each successive level. 
Dramatic, immediate, and stable improvements were seen in this case. Two weeks of baseline showed 0 occasions of sleeping in his own room and 14 occasions of sleeping on the floor of his parents room. Six steps were identified during treatment, which involved the child moving successively closer to his own room. The data pattern resulted in a meaningful and stable trend line as the child moved successfully and successively closer to the ultimate goal. The child demonstrated immediate effects, with perfect performance at each level. Likewise, he demonstrated no regression at a one-month follow-up.

This case study demonstrated significant and immediate effects with a very different type of behavioral problem not typically dealt with in school settings. Unfortunately, due to the consultation occurring at the end of the school year, there were no follow up data on the child's irrational fears at school.

Process Research. Some preliminary research has been conducted assessing verbal processes in conjoint behavioral consultation. Specifically, consultant and consultee statements in conjoint behavioral consultation have been compared to statements made in consultation with teachers only. The verbal behaviors were analyzed in relation to some of the unique goals of conjoint behavioral consultation (i.e., increased communication with and about family and child; shared ownership for problem identification across home and school settings; active parental involvement in problem solving process). Specifically, it was hypothesized that (a) there would be more statements about background environment and behavior setting in $\mathrm{CBC}$ than in traditional teacher-only consultation; (b) parent consultees would emit approximately equal numbers of utterances as teacher consultees; (c) the proportion of statements from the teacher would be significantly less in $\mathrm{CBC}$ interviews than in teacher-only consultation interviews; and (d) a greater proportion of consultant elicitors would be present in CBC interviews than in teacher-only interviews.

Verbatim transcripts of Conjoint Problem Identification Interviews from six cases were analyzed. The interviews were coded using the Consultation Analysis Record (CAR; Bergan \& Tombari, 1986), which allows for the categorization of each thought unit in terms of its source, content, process, and control characteristics. Means of statements made within each category by each participant were compared to those reported in previous research conducted by Martens, Lewandowski, and Houk (1989) that analyzed verbal processes in teacher-only consultation.

Results of the descriptive analysis both supported and contradicted the research hypotheses. Contrary to predictions, there was no difference found in the amount of statements made regarding background environment and behavior setting in the $\mathrm{CBC}$ transcripts as compared to teacher-only consultation transcripts. In both teacher-only consultation and $\mathrm{CBC}$ cases, the proportion of background environment verbalizations in $\mathrm{CBC}$ were minimal. Consistent with research predictions was the finding that parents were actively involved in Problem Identification Interviews. In fact, the percentage of verbalizations contributed by parents was slightly greater than that contributed by teachers in CBC interviews $(32 \%$ and $24 \%$ of the total statements were provided by parents and teachers, respec- 
tively). Their involvement was directly elicited (i.e., $50 \%$ of elicitors were directed toward the parent), and they appeared to provide a substantial portion of information. This finding supports the $\mathrm{CBC}$ objective of obtaining information from parents and promoting their active involvement in problem solving.

Also consistent with predictions, the teacher's role appeared to be different in $\mathrm{CBC}$ than in teacher-only consultation. Specifically, the teacher's verbal contribution to discussions in $\mathrm{CBC}$ was proportionately less than in consultation wherein the teacher served as the sole consultee $(24 \%$ of all statements in $\mathrm{CBC}$ were made by teachers, versus $74 \%$ in teacher-only consultation). Interestingly, teachers were found to ask proportionately more questions in $\mathrm{CBC}$ than in teacheronly consultation (they emitted $7 \%$ elicitors in $\mathrm{CBC}$ vs. $1 \%$ in teacher-only consultation), and consultants used proportionately fewer elicitors in CBC (19\%) than in teacher-only consultation (34\%). This finding suggests that teachers may perceive their role in $\mathrm{CBC}$ as one of shared responsibility for eliciting information from parents in CBC. However, it should be recognized that these data are purely descriptive, and do not imply statistically significant differences.

Finally, an unexpected finding regarding the consultant's role in $\mathrm{CBC}$ emerged from the process analysis. Specifically, consultants tended to control more of the discussion in CBC. Specifically, $56 \%$ of the statements made in CBC interviews were made by consultants, as compared to $26 \%$ in teacher-only consultation. To the extent that questions serve to direct interviews, consultants appear to engage in a more directive role in $\mathrm{CBC}$ than they do in consultation with teachers only. Of all elicitors in the CBC interviews, $79 \%$ were made by the consultant (similar data are unavailable for teacher-only consultation interviews). It may be that the added complexity of a second consultee (i.e., parent) encouraged consultants to make deliberate and active attempts to structure the discussion.

Acceptability Research. An important issue pertaining to the application of conjoint behavioral consultation is its acceptability in practice. A national survey of Nationally Certified School Psychologists investigating the acceptability of CBC by school psychologists was completed (Sheridan \& Steck, 1995). Using items from the Behavioral Intervention Rating Scale, the acceptability of CBC in relation to consultant variables (age, level of training, years experience, theoretical orientation, age of student served) and perceived logistical barriers (time, administrative support) was investigated. Also assessed was the differential acceptability of $\mathrm{CBC}$ in contrast to other modes of service delivery (direct service and teacher- and parent-only consultation) for academic, behavioral and social-emotional problems.

The results of the survey were very supportive of $\mathrm{CBC}$ as a consultation service delivery model. School psychologists rated $\mathrm{CBC}$ as very acceptable (mean $=4.2$ on a 5-point acceptability Likert scale). Their ratings of the acceptability of $\mathrm{CBC}$ was most affected by external constraints of time concerns and perceived administrative/organizational support for implementing the procedure. Age of students served, theoretical orientation, years of experience, and level of training had little if any impact on the acceptability ratings. 
It was found that $\mathrm{CBC}$ was rated as more acceptable than any other mode of service delivery across problem types. Further, it was rated essentially the same across all problem types, suggesting that $\mathrm{CBC}$ was perceived as more generally applicable than had been anticipated. Another important finding was that the age of student served did not appear to influence acceptability ratings of mode of service delivery, with the exception that school psychologists who serve secondary students rated direct service and CBC approximately equally.

\section{Research Directions}

Research in conjoint behavioral consultation is accumulating, with generally positive and encouraging results. There are several areas in need of investigation to further its empirical base. First, it seems necessary at this time to systematically investigate the utility of the model with children presenting various personal characteristics. For example, the usefulness of $\mathrm{CBC}$ with children from various ethnic, educational, and socioeconomic backgrounds has not been evaluated. Colton and Sheridan's (in press) sample was comprised of families in low SES conditions, however this was not a central aspect of the study and was not controlled carefully. Given the emphasis on including all parents in the educational process (including those from different demographic groups), it is necessary to determine those practices that are more or less effective with diverse families.

A second research direction should focus on assessing systematically relationship variables in conjoint behavioral consultation. For example, research that evaluates the short-term and long-term effects of conjoint problem solving on the home-school relationship could be conducted. Specific research on the generalization of the parent-teacher relationship over time is a particular interest in light of the goals of CBC. Likewise, most participants in previous research have demonstrated a willingness to engage in the process. Process and outcome researchers should begin to investigate consultation sessions under conditions where consultees have a history of interpersonal problems, lack the motivation to work together, or exhibit resistance to the consultative process.

An additional research direction concerns alternative parent, teacher, and child roles in conjoint consultation. Previous process research suggested that the teacher's role in CBC may be qualitatively different than in other forms of teacher-only consultation. It is possible that teachers could be trained to serve as a consultant with the parent and allow consultant-specialists to transition more quickly out of certain consultation sessions. Likewise, it is possible that not all parents and teachers will be able to adopt a high level of participation (i.e., ongoing consultation interviews and meetings). Procedures that allow consultants to determine the essential points at which all participants must be involved, and points at which progress can be made with only a teacher or parent present would be necessary. Finally, some research (e.g., Galloway \& Sheridan, 1994) included children in the final phase of the Problem Analysis Interview and Treatment 
Evaluation Interview to involve them in plan specification, to help them understand intervention procedures, and to assess their perceptions of outcomes. Alternative roles for clients, including their active involvement in various aspects of the consultation sessions, may be an interesting line of inquiry.

Related to issues surrounding alternative roles, there may be some desirability to identifying ways to streamline the consultation process. In anecdotal reports, consultants and consultees often state that the interviews are too lengthy and in some cases redundant. There may be circumstances wherein the interviews can be shortened. For example, some behaviors have a very stable and intractable history (e.g., selective mutism), and may not require an extensive baseline condition. In these cases, separate Problem Identification and Problem Analysis Interviews may not be necessary. Additionally, the utility of a "consultation referral form" that assists consultants and consultees identify target behavioral problems prior to entering the initial consultation interview is now being explored. Such "advanced organizers" for consultation may help focus in on target behaviors, identify previously-attempted interventions, and explore strengths of the individual.

As currently practiced, conjoint consultation services are typically offered to address presenting problems when no alternative intervention programs are in place. It may be desirable to initiate the model in cases where an alternative treatment program is in place. In these cases, the primary goal of conjoint behavioral consultation may be generalization of effects to other, nontreatment settings. For example, social skills training is typically provided in small groups to four or more students. A major limitation of many social skills programs is their inability to demonstrate generalization of learned social skills to the natural environment (DuPaul $\&$ Eckert, 1994). Conjoint behavioral consultation may be particularly relevant in encouraging students' social skills use to the classroom and home settings.

Another research direction concerns furthering the investigation of processes within $\mathrm{CBC}$. For example, a preliminary study of $\mathrm{CBC}$ verbal processes was conducted with only a limited number of Problem Identification Interviews of successful cases. The findings of this preliminary analysis suggested that, among other things, consultants seem to direct the interview actively by eliciting information, and they "control" the interview by making a proportionately larger number of verbalizations than the parent or teacher consultees. All consultation cases evaluated had positive outcomes in terms of child outcome, consumer satisfaction, and social validity; therefore, it may be necessary for consultants to engage in such behaviors to produce such results. However, other unexamined processes may also be operative and in need of analysis prior to drawing conclusions about control and directedness. It is necessary to replicate the study using a larger sample. Likewise, it is necessary to evaluate transcripts of cases where consultation goals were not met, outcomes were limited, or consultee resistance was a factor. Of importance is the need to evaluate whether consultants can remain "in control" and still "collaborative," and other skills used by consultants that may affect case outcomes. 


\section{CONCLUSIONS}

Conjoint behavioral consultation is a service delivery model that builds on the empirically-validated structure of behavioral consultation embedded within a philosophy of strengthening home-school relations and partnerships. CBC provides one structured model by which effective and meaningful partnerships with families can be established and maintained. Outcome, process, and acceptability research conducted to date suggest that the model can contribute to effective and acceptable results for children, parents, and teachers. Likewise, differences in consultant and consultee behaviors appear to exist between CBC and more traditional approaches to consultation, suggesting unique features of the model. There continues to be many areas in need of exploration, including practice and process issues within CBC. It is hoped that through continued empirical scrutiny, we may ultimately identify those families and children for whom CBC produces optimal outcomes, and the consultant practices that maximize consultation effects.

\section{REFERENCES}

Apter, S. J. (1982). Troubled children/troubled systems. Elmsford, NY: Pergamon Press.

Bergan, J. R., \& Kratochwill, T. R. (1990). Behavioral consultation in applied settings. New York: Plenum.

Bergan, J. R., \& Tombari, M. L. (1976). Consultant skill and efficiency and the implementation and outcomes of consultation. Journal of School Psychology, 14, 3-14.

Christenson, S. L. (1995). Families and schools: What is the role of the school psychologist? School Psychology Quarterly, 10,118-132.

Colton, D. L., \& Sheridan, S. M. (in press). Conjoint behavioral consultation and social skills training: Enhancing the play behaviors of boys with attention deficit-hyperactivity disorder. Journal of Educational and Psychological Consultation.

Conoley, J. C. (1987). Strategic family intervention: Three cases of school-aged children. School Psychology Review, 16, 469-486.

Drabman, R. S., Hammer, D., \& Rosenbaum, M. S. (1979). Assessing generalization in behavior modification with children: The generalization map. Behavioral Assessment, 1, 203-219.

DuPaul, G., \& Eckert, T. (1994). The effects of social skills curricula: Now you see them, now you don't. School Psychology Quarterly, 9, 113-132.

Galloway, J., \& Sheridan, S. M. (1994). Implementing scientific practices through case studies: Examples using home-school interventions and consultation. Journal of School Psychology, 32, 385-413.

Gresham, F. M, \& Elliott, S. N. (1990). The Social Skills Rating System. Circle Pines, MN: AGS. Hansen, D. A. (1986). Family-school articulations: The effects of interaction rule mismatch. American Educational Research Journal, 23, 643-659.

Kazdin, A. E. (1977). Assessing the clinical or applied importance of behavior change through social validation. Behavior Modification, 1, 427-452.

Kratochwill, T. R., \& Bergan, J. R. (1990). Behavioral consultation in applied settings: An individual guide. New York: Plenum. 
Martens, B. K., Lewandowski, L. J., \& Houk, J. L. (1989). Correlational analysis of verbal interactions during the consultative interview and consultees' subsequent perceptions. Professional Psychology: Research and Practice, 20, 334-339.

McGinnis, E., \& Goldstein, A. P. (1984). Skillstreaming the elementary school child: A guide for teaching prosocial skills. Champaign, IL: Research Press.

Sheridan, S. M, \& Colton, D. L. (1994). Conjoint behavioral consultation: A review and case study. Journal of Educational and Psychological Consultation, 5, 211-228.

Sheridan, S. M., \& Kratochwill, T. R. (1992). Behavioral parent-teacher consultation: Conceptual and research considerations. Journal of School Psychology, 30, 117-139.

Sheridan, S. M., Kratochwill, T. R., \& Bergan, J. R. (1996). Conjoint Behavioral Consultation: A procedural manual. New York: Plenum.

Sheridan, S. M., Kratochwill, T. R., \& Elliott, S. N. (1990). Behavioral consultation with parents and teachers: Applications with socially withdrawn children. School Psychology Review, 19,33-52.

Sheridan, S. M., \& Steck, M. (1995). Acceptability of conjoint behavioral consultation: A national survey of school psychologists. School Psychology Review.

Von Brock, M. B., \& Elliott, S. N. (1987). Influence of treatment effectiveness information on the acceptability of classroom interventions. Journal of School Psychology, 25, 131-144.

Wahler, R. G., \& Fox, J. J. (1981). Setting events in applied behavior analysis: Toward a conceptual and methodological expansion. Journal of Applied Behavior Analysis, 14, $327-338$.

Witt, J. C, \& Elliott, S. N. (1985). Acceptability of classroom intervention strategies. In T. R. Kratochwill (Ed.), Advances in school psychology (Vol. 4, pp. 251-288). Hillsdale, NJ: Lawrence Erlbaum. 